Proceedings of the 31st Annual Meeting of the Brazilian Embryo Technology Society (SBTE); Cabo de Santo Agostinho, PE, Brazil, August 17th to 19th, 2017.

\title{
Cryopreservation of in vitro-produced embryos: challenges for commercial implementation
}

\author{
Bruno Valente Sanches ${ }^{1}$, Amanda Fonseca Zangirolamo ${ }^{1}$, Nathalia Covre da Silva ${ }^{1}$, Fabio Morotti ${ }^{1}$, \\ Marcelo Marcondes Seneda ${ }^{2,3}$ \\ ${ }^{1}$ Cogent IVF, LLC, Hermiston, OR 97838, USA. \\ ${ }^{2}$ Laboratório de Reprodução Animal, DCV-CCA-UEL, Londrina, PR, Brazil.
}

\begin{abstract}
In the last several years, the high demand for embryo production has resulted in the need to study new methods to make the cryopreservation of bovine embryos produced in vitro more efficient. Despite the advantages offered by in vitro embryo production (IVEP), the major challenge to its greater dissemination is to improve embryonic survival after cryopreservation. Embryos that are produced in vitro are less resistant to cryopreservation compared to those produced in vivo, which is due to the higher accumulation of lipids in their cells, among other factors. In this context, changes in the culture conditions such as the addition of lipolytic chemical substances and the adjustment of fetal calf serum in the medium have been proposed to decrease the lipid amount within the embryos. Several years ago, vitrification allowed good results for in vitro produced (IVP) embryos because of its simplicity, speed and low cost. More recently, another technique applied to simplify the embryo post-thawing rehydration step in vivo, direct transfer (DT), is a strategy that has proven to be of interest in helping to overcome limitations to the cryopreservation of in vitro produced embryos. DT has been performed by commercial laboratories, ensuring good embryo viability after thawing. However, commercial and operational limitations prevent the large-scale use of these techniques. Thus, this review aims to discuss the use of strategies to improve the postcryopreservation survival capacity and the aspects to be overcome so that the cryopreservation of IVP embryos becomes a well-established and commercially applicable technique in addition to presenting new guidelines for embryo transfer (ET) programs from a better selection of recipients.
\end{abstract}

Keywords: bovine, commercial limitation, cryopreservation, in vitro-produced embryos, recipient cow.

\section{Introduction}

During the year 2015, almost 700.000 IVP embryos were produced, surpassing for the first time the number of bovine embryos produced in vivo. In this context, 269.353 bovine OPU IVP embryos were transferred in Brazil alone (Perry, 2016), which is considered the world's largest producer of bovine embryos. This situation is directly related to the predominance of Bos indicus cattle. Several studies have reported that Zebu females, when submitted to ovum pick-up (OPU) guided by transvaginal ultrasonography, had a higher number of oocytes aspirated than Bos taurus females (Segerson et al., 1984; Silva-Santos et al., 2011). This feature favors large-scale in vitro embryo production (IVEP) in both dairy and beef cattle (Pontes et al., 2011).

Furthermore, IVEP has advantageous conditions for its application in Bos indicus dairy cattle, since these animals, in addition to being good donors of oocytes, are adapted to a tropical climate and can produce milk even under high-temperature conditions (Marinho et al., 2015). Another advantage is the fact that embryos are more resistant than gametes when subjected to high body temperatures due to thermal stress (Chebel et al., 2008). Thus, the pregnancy rates are better in embryo transfer (ET) than artificial insemination (AI) throughout the year (Stewart et al., 2011; Ferreira, 2013).

Additionally, in the last decade, there has been a significant increase in the production of sexed embryos, especially due to the search for genetic improvement of dairy cattle (Pontes et al., 2010). Another advantage of IVEP compared with in vivo methods is the smaller number of viable sperm required for fertilization and, therefore, more efficient results in the use of sex-sorted semen (Pontes et al., 2010; Morotti et al., 2014).

In this context, the total embryo production is sometimes higher than the number of embryos transferred, so investment in research was increased to develop an efficient protocol for the cryopreservation of the remaining embryos in a program (Sanches et al., 2016). Despite the advantages provided by IVEP, the greatest challenge of this biotechnology is the lower resistance to the cryopreservation process that these embryos present (Sudano et al., 2011).

The high sensitivity to cooling of in vitro embryos is reported to be due to the greater accumulation of lipids in their cells (Abe et al., 2002), arranged in the form of cytoplasmic lipid droplets that are constituted predominantly of triglycerides (McKeegan and Sturmey, 2012). Additionally, there are indications that this high lipid content is because of the medium in which the embryos are cultured (Abe et al., 2002; Sanches et al., 2013). Thus, some strategies for improving post-cryopreservation survival capacity have been studied and tested to produce more cryotolerant embryos (Sudano et al., 2013).

Among cryopreservation techniques, vitrification 
has been used worldwide (Dode et al., 2013) because of its simplicity, speed, and low cost. However, this technique requires a high concentration of cryoprotectants in addition to a trained person to perform a morphological evaluation of embryo quality before the loading process (Vajta et al., 1998).

In contrast, Sanches et al. (2016) demonstrated that a technique used since the 1990s to simplify the post-thawing rehydration step of in vivo embryos direct transfer (DT) - can also be used for frozen in vitro embryos. The DT strategy has been demonstrated to be helpful for overcoming limitations to in vitro embryo cryopreservation, since it has been recently performed by commercial laboratories, providing good embryo viability after thawing.

The choice of embryo recipients is another important step in the implementation of IVEP programs (Peixoto et al., 2004). Age, sanitary and nutritional conditions, such as synchrony between the recipients and embryonic stage, are important attributes to take into account in the choice of embryo recipients (Hasler et al., 1987; Sreenan and Diskin, 1987; Callesen et al., 1996; Jones and Lamb, 2008). Moreover, recent strategies for the synchronization of estrus/ovulation and the selection of recipients by fertility have been achieved (Marinho et al., 2012).

In contrast, there are still many commercial and operational limitations of bovine IVEP embryos and cryopreservation processes, which prevent its use on a large scale. Examples include the need for a qualified person to perform all stages of IVEP and the cryopreservation process, logistics between laboratory and recipients, as well as a trained technician in the field due the particularities of warming those cryopreserved embryos before transfer (Hasler, 2010; Saragusty and Arav, 2011).

Therefore, considering the importance of implementing an efficient IVEP and cryopreservation program, this review aims to discuss i) the use of strategies to improve embryo post-cryopreservation capacity; ii) the choice of recipients with good sanitary/nutritional conditions and reproductive characteristics to maintain a healthy pregnancy; and finally, iii) a team able to perform all stages of IVEP with rigorous quality control and the logistics necessary for making ET feasible in the field.

\section{Cryopreservation of bovine embryos}

Methods and differences of cryotolerance in embryos produced in vivo and in vitro

The process of embryo cryopreservation is the most challenging aspect of embryo biotechnology, and despite advances in recent years, the results are still inconsistent (Sudano et al., 2013). During embryo freezing, the cryopreservation method aims to avoid the formation of intracellular ice crystals and to decrease the toxic effects generated by the cryoprotectant agent, minimizing the osmotic stress to the cells (Pryor et al., 2009).

Cryopreservation protocols are based on two variables: type and concentration of cryoprotectant and cooling rates (Vajta and Kuwayama, 2006). Currently, slow freezing (classic) and vitrification (ultra-rapid) are the two main methods used commercially for IVEP embryo cryopreservation (Saragusty and Arav, 2011).

Vitrification is the predominant technique used for IVEP (Dode et al., 2013) due to being a simple, fast and low-cost method (Sanches et al., 2016). In this method, a high-osmolarity solution is used so that the embryonic intracellular water exits rapidly, dehydrating the embryonic cells and making them permeable to the cryoprotectant. Thus, the embryo is able to withstand direct immersion in liquid nitrogen $\left(-196^{\circ} \mathrm{C}\right)$ without the formation of ice crystals (Vajta et al., 1998).

On the other hand, high cryoprotectant concentrations have been described as promoting high cellular toxicity, even if exposed for a short period and a minimum volume of this solution (Vajta et al., 1998). Thus, different strategies have been developed for embryos to have rapid contact with liquid nitrogen and to reduce the volume of the cryoprotectant agent, such as the open pulled straw (OPS; Vajta et al., 1998), cryoloop (Lane et al., 1999), microdroplets (Papis et al., 2000) and cryotop techniques (Kuwayama et al., 2005).

In the classical slow-freezing protocol, the cooling rate is controlled to maintain a constant curve until the straws with embryos are immersed in the liquid nitrogen. The use of low concentrations of cryoprotectants is the main advantage of this technique since high concentrations are toxic to embryos. In addition, the process of thawing and the DT of embryos to cows make the slow freezing protocol more efficient for commercial use.

However, ice crystals can form and damage the structure of the embryo's membranes and organelles (Dode et al., 2013). In this way, the success of slow freezing and direct transfer of in vitro produced embryos invariably depends on the equilibrium between the rate of dehydration of the cell and the rate at which water is transformed into ice crystals (Visintin et al., 2002).

Despite the advances in cryopreservation methods, freezing and thawing processes impair the viability of the embryo. This impairment occurs due to the physical and chemical damages induced during the cryopreservation process (Overstrom, 1996; Baguisi et al., 2000). Sudano et al. (2012a) reported the effects of this damage by comparing the apoptosis rate caused by the stress of cryopreservation between fresh and vitrified blastocysts. In this study, there was a 2.4-fold increase $(\mathrm{P}<0.0001)$ in the apoptosis rate of vitrified $(49.4 \pm 1.9)$ in relation to fresh embryos $(20.8 \pm 1.1)$. Similar apoptosis profiles were observed in other studies, which demonstrated increases of 3.7-fold (Park et al., 2006) and 1.7-fold (Márquez-Alvarado et al., 2004 ) in the apoptosis rate of cryopreserved embryos compared with fresh embryos.

Moreover, it has been definitively demonstrated that in vitro embryos are more sensitive to cryopreservation than in vivo embryos (Pollard and Leibo, 1994). This lower cryotolerance has been associated with the high lipid content present in the 
cytoplasm of these embryos (Abe et al., 2002; Mucci et al., 2006) and the decrease in the density of mature mitochondria compared to embryos produced in vivo (Crosier et al., 2001; Farin et al., 2004). Additionally, the most abundant lipids in the plasma membranes of cells (phosphatidylcholine and sphingomyelin) also have different profiles (Sudano et al., 2012b).

Researchers suggest that lipid accumulation may be due to the uptake of the culture medium itself or to the inefficient and unregulated metabolism of the embryonic mitochondria (Farin et al., 2004; Barceló-Fimbres and Seidel, 2007a; Moore et al., 2007). Further, in vitro embryos have fewer transcripts levels for genes related to lipid metabolism compared to in vivo-produced embryos (Gad et al., 2012). Therefore, the addition of substances to the culture medium has been proposed in addition to adjusting the cryopreservation method to make the embryos more cryotolerant (Dode et al., 2013).

Strategies to increase the cryotolerance of in vitro embryos

Despite many advances in the last decades, the cryopreservation process of IVEP remains a major challenge in livestock, and the results are still inconsistent (Sudano et al., 2013). For example, the low cryotolerance of in vitro embryos is the main obstacle to the use of cryopreservation protocols (Sudano et al., 2011). The role of embryonic lipids in this regard is well described in the literature (Abe et al., 2002). Furthermore, strategies such as the use of serum-free culture media, the addition of chemical substances to promote changes in lipid metabolism, and the modulation of the membrane lipid composition can help improve the survival of in vitro embryos after cryopreservation (Sudano et al., 2013).

The cause of cytoplasmatic lipid deposition in in vitro embryos is not well established, but it has been suggested that the presence of serum in the culture medium may be directly involved in this process (Sanches et al., 2013). Studies have shown that the fetal calf serum (FCS) concentration affects the number of cytoplasmic lipid droplets of embryos (Leroy et al., 2005; Sudano et al., 2012a). Moreover, in vitro embryos cultured in a serum-free medium had decreased lipids and higher cryotolerance (Pereira and Marques, 2008).

An alternative to improving embryo freezeability is the use of lipolytic chemical agents, such as phenazine ethosulfate (PES), which reduces lipid accumulation and regulates energetic metabolism by NADPH to NADP oxidation (De La Torre-Sanchez et al., 2006; Sudano et al., 2011). Interestingly, it has been reported that PES, when used in the post-compaction period, promoted an increase in post-cryopreservation survival (Barceló- Fimbres and Seidel, 2007b).

In this sense, a study involving supplementation with FCS and PES showed an improvement in the blastocoele re-expansion rate after the embryo vitrification process when the serum concentration was reduced to $2.5 \%$ concomitant to the addition of PES to the culture medium on day 4 (Table 1; Sudano et al., 2011).

Table 1. Effects of fetal calf serum (FCS) and phenazine ethosulfate (PES) on blastocoele re-expansion (means \pm SEM).

\begin{tabular}{|c|c|c|}
\hline \multirow{2}{*}{ Responses } & \multicolumn{2}{|c|}{ Cryotolerance } \\
\hline & Vitrified embryos (n) & Re-expansion rate $(\%)$ \\
\hline \multicolumn{3}{|l|}{ FCS } \\
\hline $0 \%$ & 233 & $90.5 \pm 2.7^{\mathrm{a}}$ \\
\hline $2.5 \%$ & 346 & $81.6 \pm 2.5^{\mathrm{b}}$ \\
\hline $5 \%$ & 332 & $78.0 \pm 2.8^{\mathrm{bc}}$ \\
\hline $10 \%$ & 405 & $67.3 \pm 3.5^{\mathrm{c}}$ \\
\hline In vivo control & 15 & $93.3 \pm 6.7^{\mathrm{aA}}$ \\
\hline \multicolumn{3}{|l|}{ PES } \\
\hline Control & 474 & $72.0 \pm 3.0^{\mathrm{B}}$ \\
\hline PES day 2.5 & 362 & $79.9 \pm 2.8^{\mathrm{C}}$ \\
\hline PES day 4.0 & 480 & $86.2 \pm 2.4 \mathrm{~A}^{\mathrm{C}}$ \\
\hline
\end{tabular}

${ }^{\mathrm{a}-\mathrm{d}}$ Within a column, means without a common superscript $\operatorname{differ}(\mathrm{P}<0.05) .{ }^{\mathrm{A}-\mathrm{C}}$ Within a column, means without a common superscript differ $(\mathrm{P}<0.05)$. Adapted from Sudano et al. (2011).

It is important to note that the addition of medium with $2.5 \%$ FCS did not decrease the embryonic cryotolerance (represented by the blastocoele reexpansion rate) compared to the group without FCS. However, independent of FCS concentration in the medium and the use of PES, the embryos in the in vivo group (control) had the highest survival after vitrification (Sudano et al., 2011).

Forskolin is another lipolytic chemical agent used to reduce the lipid content of in vitro embryos. This agent acts directly by activation of the adenylate cyclase, thus increasing the levels of cAMP and stimulating lipolysis to activate lipases (Men et al., 2006). Recently, Paschoal et al. (2017) demonstrated that forskolin was an effective lipolytic agent even at low concentrations, resulting in the formation of blastocysts with a larger number of cells than the untreated group. Additionally, this substance decreased embryo apoptosis caused by the cryopreservation method.

Therefore, it has been previously reported that treatment with forskolin before vitrification with the 
cryotop method (a polypropylene rod in which the embryos are allocated next to minimum volumes of cryoprotectant solution) improved the cryotolerance and pregnancy rates of Bos indicus in vitro embryos after transfer to recipients (Sanches et al., 2013). The results are shown in Table 2.

Table 2. Pregnancy rates of Bos indicus in vitro embryos treated with or without the lipolytic agent forskolin for 48 hours in culture before the vitrification process.

\begin{tabular}{lcc}
\hline Treatment & $\begin{array}{c}\text { Transferred embryos } \\
(\mathrm{n})\end{array}$ & $\begin{array}{c}\text { Pregnancy rate } \\
(\%)\end{array}$ \\
\hline Control & 65 & $18.5^{\mathrm{b}}$ \\
Forskolin & 80 & $48.8^{\mathrm{a}}$ \\
\hline
\end{tabular}

${ }^{\mathrm{a}, \mathrm{b}}$ Within a column, rates without a common superscript differed $(\mathrm{P}<0.05)$. Adapted from Sanches et al. (2013).

According to these results, the use of forskolin and vitrification with the cryotop system as a strategic cryopreservation system could be a great alternative to facilitate the transport and export of embryos over long distances (Sanches et al., 2013).

In addition, the stage of development of blastocysts at the time they undergo cryopreservation is another factor that needs to be considered as a strategy to improve cryotolerance. For example, Kocyigit and Cevik (2016), showed a correlation between the diameter of embryos and their cryosurvival, in which early and expanded blastocysts were more sensitive to the damage promoted by vitrification and posterior warming compared to the blastocyst stage. In our experience, the ideal developmental stages are blastocyst and expanded blastocyst for both vitrification and direct transfer methods.

The slow freezing of embryos for later DT, despite having higher costs, eliminates the evaluation before transfer, which makes it more practical than vitrification. Moreover, smaller concentrations of cryoprotectants may also be used, thereby reducing toxicity to the embryos (Voelkel and $\mathrm{Hu}, 1992$ ).

Briefly, in the DT method, the in vitro embryos are cryopreserved by the slow freezing method previously described for in vivo embryos (Vajta et al., 1998). The in vitro embryos are next exposed to a freezing solution consisting of $1.5 \mathrm{M}$ ethylene glycol (EG), and at the end of the freezing curve, they are directly immersed in liquid nitrogen and stored until being transferred into the recipients.

Surprisingly, this strategy has been demonstrated to help overcome obstacles to in vitro embryo cryopreservation. On Table 3 pregnancy rates for fresh, vitrified, and frozen (direct transfer) in vitro embryos from dairy cows are presented.

Table 3. Pregnancy rates at 30 days after the transfer of fresh, vitrified or frozen (direct transfer) in vitro-produced embryos after ovum pick-up of Girolando cows.

\begin{tabular}{lcc}
\hline Group & $\begin{array}{c}\text { Transferred embryos } \\
(\mathrm{n})\end{array}$ & $\begin{array}{c}\text { Pregnancy } \\
(\%)\end{array}$ \\
\hline Fresh & 259 & $43.24 \pm 1.23^{\mathrm{a}}$ \\
Vitrified & 234 & $31.19 \pm 4.01^{\mathrm{b}}$ \\
Frozen & 311 & $34.72 \pm 4.15^{\mathrm{b}}$ \\
\hline
\end{tabular}

${ }^{\mathrm{a}, \mathrm{b}}$ Different letters in the same column indicate a significant difference $(\mathrm{P}<0.05)$. Adapted from Sanches et al. (2016).

The results of this study revealed the possibility of using frozen embryos because the direct transfer optimized the logistics and can become a more practical approach for the transfer of cryopreserved in vitro embryos in the field (Sanches et al., 2016).

The direct transfer protocol has been used in large-scale operations, especially in the US and Brazil. In the near future, once other companies incorporate the direct transfer protocol in their operation, the majority of commercial IVEP embryos will probably be frozen, as currently occurs in the semen industry.

Despite the advances in cryopreservation methods, few players are using this technique, and some challenges remain in relation to the greater efficiency of the technique.

\section{Importance of recipient cow selection}

The choice of recipients is an important part of the success of bovine ET programs, since many of problems with this biotechnology application are related to the female conditions that will allow embryo implantation and maintenance of gestation until the fetus is born (Andrade et al., 2012).

Among the factors that directly interfere with the performance of fresh or cryopreserved transferred embryos, major highlighted aspects are the recipient's age, the sanitary and nutritional conditions of the recipients, and the degree of synchrony between the embryo stage and its recipient (Sreenan and Diskin, 1987; Hasler et al., 1987; Callesen et al., 1996; Peixoto et al., 2004, Jones and Lamb, 2008).

An interesting study evaluated the effects of synchrony between embryo stage and recipient on conceptus elongation and pregnancy rate. In this study, the authors showed that conceptus length was greater following transfer to an advanced uterus and that supplementation with progesterone resulted in short cycles in approximately $50 \%$ of recipients. Transfer of day 7 embryos to a synchronous uterus (day 7) resulted in a pregnancy rate of $47.3 \%$. Transfer to an asynchronous uterus of day $5(40.8 \%)$ or day 8 
embryos $(41.3 \%)$ moderately impacted the pregnancy rate $(\mathrm{P}<0.01)$, but transfer to the uterus 2 days in advance (day 9, 24.4\%) or 3 days behind (day 4, 27.0\%) reduced $(\mathrm{P}<0.001)$ the pregnancy rate compared with synchronous transfer (Randi et al., 2015). Interestingly, this study emphasized the importance of greater possible synchrony between the embryonic stage and the cycle day of the recipient.

Additionally, new technologies have been developed with the aim of helping the selection of recipients be more accurate by searching for genetic markers related to desirable characteristics. The sequencing of the bovine genome allowed genome-wide association studies (GWAS) to be conducted, which examine specific sites, such as single-nucleotide polymorphisms (SNPs), and associate them with certain phenotypes (Dairy Herd Management, 2017).

Recently, it has been possible to use commercial programs aimed at the identification of genomic loci associated with fertility in heifers and dairy cows. Some specific genes associated with fetal abnormalities that lead to abortion, embryonic death, or lower fertility, as well as genes associated with better reproductive efficiency, have been identified.

Therefore, this information associated with other methods and criteria for choosing the recipient can help the optimization and practical success of ET and consequently can improve the efficiency of IVEP in the field.

\section{Commercial and operational limitations to cryopreservation of in vitro-produced embryos}

Cryopreservation of bovine embryos is a biotechnology that allows the storage of surplus embryos produced in vitro or through superovulation/embryo transfer programs, making feasible commercialization between countries and the transfer of embryos at a more convenient time (Sudano et al., 2012b).

However, the number of embryos cryopreserved in the past several years represented only 3 to $7 \%$ of the total embryo production in Brazil (Stroud, 2011, 2012; Viana, 2012). These data reflect the great challenges to the implementation of this technique.

As previously discussed, the low cryotolerance of in vitro embryos is a crucial obstacle to the use of cryopreservation processes in IVEP programs (Sudano et al., 2011). In this context, many efforts have been made by different research groups to improve the culture medium conditions during IVEP or to change cryopreservation protocols (Sudano et al., 2013). It is also important to emphasize that the survival capacity of the embryo after cryopreservation is a multifactorial event (Sudano et al., 2013).

Embryonic survival after freezing/thawing is influenced by important aspects, such as the culture medium composition (additives, supplementation with or without fetal calf serum, $\mathrm{pH}$, and osmolarity), oocyte and semen quality, and the technician who produced the embryo in the laboratory (Gardner, 2008; Feugang et al., 2009; Hasler, 2010). Another feature to be considered is the atmosphere (lower or higher oxygen tension) in which the embryos are grown, which has been widely used to minimize oxidative stress; low oxygen tension improves metabolism and decreases the production of free radicals (Dode et al., 2013).

Finally, the qualification of the field veterinarian/technical responsible for performing the embryo transfer into the recipient uterus is another factor limiting the use of cryopreserved embryos in ET programs. In general, the professional must perform the process in a careful, rapid and accurate manner.

In our experience, this job position (embryo transfer) will be the next limitation to using IVEP cryopreservation on a large scale and globally. Once the technology is proven and well accepted, there will not be a sufficient number of field technicians able to perform embryo transfers.

Therefore, these factors, when considered together, will directly reflect pregnancy rates and may have positive impacts on the large-scale application of IVEP and embryo cryopreservation in cattle.

\section{Final comments}

In the last decade, several technical advances have increased the efficiency of IVEP, making this reproductive strategy to have a greater impact on selection and genetic dissemination in cattle. On the other hand, the need for an efficient method to cryopreserve the surplus volume from embryo programs was generated. For in vitro embryos, vitrification has become the most frequently used technique for cryopreservation worldwide, which has contributed widely to the storage of embryos, as well as making IVEP programs more efficient.

Therefore, the implementation of a commercial program for IVEP and cryopreservation needs to overcome many challenges when using a vitrification protocol. There is no question that we must develop and improve the efficiency of direct transfer techniques to make the IVEP technology accessible to everyone everywhere. Along with the selection of the recipient according to good sanitary and nutritional status, adequate synchrony between embryo stage and recipient cycle, high maternal ability, and the choice of females with characteristics linked to fertility are aspects fundamental to the success of this biotechnology. Finally, the entire in vitro process for production or cryopreservation requires a highly qualified and trained team to perform each step of this journey.

\section{References}

Abe H, Yamashita S, Satoh T, Hoshi H. 2002. Accumulation of cytoplasmic lipid droplets in bovine embryos and cryotolerance of embryos developed in different culture systems using serum-free or serumcontaining media. Mol Reprod, 61:57-66.

Andrade GA, Fernandes MA, Knychala RM, Pereira Junior MV, Oliveira AJ, Nunes DP, Bonato G, Santos RM. 2012. Fatores que afetam a taxa de prenhez de receptoras de embriões bovinos produzidos in vitro. Rev Bras Reprod Anim, 36:66-69.

Baguisi A, Lonergan P, Overstrom E, Boland $M$. 
2000. Vitrification of bovine embryos: incidence of necrosis and apoptosis. Theriogenology, 55:162.Abstract. Barceló-Fimbres M, Seidel GEJ. 2007a. Effects of either glucose or fructose and metabolic regulators on bovine embryo development and lipid accumulation in vitro. Mol Reprod Dev, 74:1406-1418.

Barceló-Fimbres M, Seidel GEJ. 2007b. Effects of fetal calf serum, phenazine ethosulfate and either glucose or fructose during in vitro culture of bovine embryos on embryonic development after cryopreservation. Mol Reprod Dev, 74:1395-1405.

Callesen H, Liboriussen T, Greve T. 1996. Practical aspects of multiple ovulation-embryo transfer in cattle. Anim Reprod Sci, 42:205-214.

Chebel RC, Demétrio DGB, Metzger J. 2008. Factor affecting success of embryo collection and transfer in large dairy herds. Theriogenology, 69: 98-106.

Crosier AE, Farin PW, Dykstra MJ, Alexander JE, Farin CE. 2001. Ultrastructural morphometry of bovine blastocysts produced in vivo or in vitro. Biol Reprod, 64:1375-1385.

Dairy Herd Management [homepage on the Internet] 2017. The genomics of fertility and health traits Available on: http://www.dairyherd.com/advice-andtips/reproduction/genomics-fertility-and-health-traits.

Accessed on: April 10, 2017.

De La Torre-Sanchez JF, Gardner DK, Preis K, Gibbons J, Seidel GE. 2006. Metabolic regulation of in vitro-produced bovine embryos. II. Effects of phenazine ethosulfate, sodium azide and 2,4-dinitrophenol during post-compaction development on glucose metabolism and lipid accumulation. Reprod Fertil Dev, 18:597-607.

Dode MAN, Leme LO, Spricigo JFW. 2013. Criopreservação de embriões bovinos produzidos in vitro. Rev Bras Reprod Anim, 37:145-150.

Farin CE, Farin PW, Piedrahita JA. 2004. Development of fetuses from in vitro-produced and cloned bovine embryos. J Anim Sci, 82(E-suppl):53-62.

Ferreira G. 2013. Reproductive performance of dairy farms in western Buenos Aires province, Argentina. $J$ Dairy Sci, 96:8075-8080.

Feugang JM, Camargo-Rodriguez O, Memili E 2009. Culture systems for bovine embryos. Livest Sci, 121:141-149.

Gad A, Hoelker M, Besenfelder U, Havlicek V, Cinar U, Rings F, Held E, Dufort I, Sirard MA, Schellander K, Tesfaye D. 2012. Molecular mechanisms and pathways involved in bovine embryonic genome activation and their regulation by alternative in vivo and in vitro culture conditions. Biol Reprod, 87:100. doi: 10.1095/biolreprod.112.099697.

Gardner DK. 2008. Dissection of culture media for embryos: the most important and less important components and characteristics. Reprod Fertil Dev, 20:9-18.

Hasler JF, Mccauley AD, Lathrop WF, Foote RH. 1987. Effect of donor-embryo-recipient interactions on pregnancy rate in a large-scale bovine embryo transfer program. Theriogenology, 27:139-168.

Hasler JF. 2010. Synthetic media for culture, freezing and vitrification of bovine embryos. Reprod Fertil Dev, 22:119-125
Jones AL, Lamb GC. 2008. Nutrition, synchronization, and management of beef embryo transfer recipients. Theriogenology, 69:107-115.

Kocyigit A, Cevik M. 2016. Correlation between the cryosurvival, cell number and diameter in bovine in vitro produced embryos. Cryobiology, 73: 203-208.

Kuwayama M, Vajta G, Kato O, Leibo SP. 2005. Highly efficient vitrification method for cryopreservation of human oocytes. Reprod Biomed, 11:300-308.

Lane M, Schoolcraft WB, Gardner DK. 1999. Vitrification of mouse and human blastocysts using a novel cryoloop container-less technique. Fertil Steril, 72:1073-1078

Leroy JL, Opsomer G, De Vliegher S, Vanholder T, Goossens L, Geldhof A, Bols PE, DE Kruif A, Van Soom A. 2005. Comparison of embryo quality in highyielding dairy cows, in dairy heifers and in beef cows. Theriogenology, 64:2022-2036.

Marinho LSR, Untura RM, Morotti F, Moino LL, Rigo AG, Sanches BV, Pontes JHF, Seneda MM. 2012. Large-scale programs for recipients of in vitroproduced embryos. Anim Reprod, 9:323-328.

Marinho LSR, Sanches BV, Rosa CO, Tannura JH, Rigo AG, Basso AC, Pontes JHF, Seneda MM. 2015. Pregnancy rates to fixed embryo transfer of vitrified IVP Bos indicus, Bos Taurus or Bos indicus $x$ Bos taurus embryos. Reprod Domest Anim, 50:807-811.

Márquez-Alvarado YC, Galina CS, Castilla B, León H, Moreno-Mendoza N. 2004. Evidence of damage in cryopreserved and fresh bovine embryos using the TUNEL technique. Reprod Domest Anim, 39:141-145.

McKeegan PJ, Sturmey RG. 2012. The role of fatty acids in oocyte and early embryo development. Reprod Fertil Dev, 24:59-67.

Men H, Agca Y, Riley LK, Critser JK. 2006. Improved survival of vitrified porcine embryos after partial delipation through chemically stimulated lipolysis and inhibition of apoptosis. Theriogenology, 66:2008-2016

Moore K, Rodriguez-Sallaberry CJ, Kramer JM, Johnson S, Wroclawska,E, Goicoa S, Niasari-Naslaji A. 2007. In vitro production of bovine embryos in medium supplemented with a serum replacer: effects on blastocyst development, cryotolerance and survival to term. Theriogenology, 68:1316-1325.

Morotti F, Sanches BV, Pontes JHF, Basso AC, Siqueira ER, Lisboa LA, Seneda MM. 2014. Pregnancy rate and birth rate of calves from a largescale IVF program using reverse-sorted semen in Bos indicus, Bos indicus-taurus, and Bos taurus cattle. Theriogenology, 81:696-701.

Mucci N, Aller J, Kaiser GG, Hozbor F, Cabodevila J, Alberio RH. 2006. Effect of estrous cow serum during bovine embryo culture on blastocyst development and cryotolerance after slow freezing or vitrification. Theriogenology, 65:1551-1562.

Overstrom EW. 1996. In vitro assessment of embryo viability. Theriogenology, 45:3-16.

Papis K, Shimizu M, Izaike Y. 2000. Factors affecting the survivability of bovine oocytes vitrified in droplets. Theriogenology, 54:651-658. 
Park SY, Kim EY, Cui XS, Tae JC, Lee WD, Kim NH, Park SP, Lim JH. 2006. Increase in DNA fragmentation and apoptosis-related gene expression in frozen-thawed bovine blastocysts. Zygote, 14:125-131. Paschoal DM, Sudano MJ, Schwarz KRL, Maziero RRD, Guastali MD, Crocomo LF, Magalhães LCO, Martins Jr, A, Leal ALV, Landim-Alvarenga FC. 2017. Cell apoptosis and lipid content of in vitroproduced, vitrified bovine embryos treated with forskolin. Theriogenology, 87:108-114.

Peixoto MGCD, Bergmann JAG, Alvim MTT, Penna VM. 2004. Fatores que influenciaram a prenhez de embriões zebuínos em receptoras mestiças. In: Abstracts of the V Simpósio da Sociedade Brasileira de Melhoramento Animal, 2004, Pirassununga, SP. Pirassununga, SP: SBMA. 4 pp.

Pereira RM, Marques CC. 2008. Animal oocyte and embryo cryopreservation. Cell Tissue Bank, 9:267-277.

Perry G. 2016. IETS 2016. 2015 Statistics of embryo collection and transfer in domestic farm animals. Embryo Technology Newsletter, 34: 1-75.

Pollard JW, Leibo SP. 1994. Chilling sensitivity of mammalian embryos. Theriogenology, 41:101-106.

Pontes JHF, Silva KCF, Basso AC, Rigo AG, Ferreira CR, Santos GMG, Sanches, BV, Porcionato, JPF, Vieira, PHS, Faifer, FS, Sterza, FAM, Schenkd, JL, Seneda, MM. 2010. Large-scale in vitro embryo production and pregnancy rates from Bos taurus, Bos indicus, and indicus-taurus dairy cows using sexed sperm. Theriogenology, 74:1349-1355.

Pontes JHF, Melo Sterza FA, Basso AC, Ferreira CR, Sanches BV, Rubin KC, Seneda MM. 2011 Ovum pick up, in vitro embryo production, and pregnancy rates from a large-scale commercial program using Nelore cattle (Bos indicus) donors. Theriogenology, 75:1640-1646.

Pryor JH, Trant JA, Ponchirolli-Schneider CB, Looney CR, Long CR, Forrest DW. 2009. The use of forskolin and its effect on in vitro-produced brahmansired embryos submitted to slow cool freezing or vitrification. Reprod Fertil Dev, 22:214-214.

Randi F, Fernandez-Fuertes B, McDonald M, Forde N, Kelly AK, Amorin HB, Lima EM, Morotti F, Seneda MM, Lonergan P. 2015. Asynchronous embryo transfer as a tool to understand embryo-uterine interaction in cattle: is a large conceptus a good thing? Reprod Fertil Dev, 28:1999-2006.

Sanches BV, Marinho LSR, Filho BDO, Pontes JHF, Basso AC, Meirinhos, MLG, Silva-Santos KC, Ferreira CR, Seneda MM. 2013. Cryosurvival and pregnancy rates after exposure of IVF-derived Bos indicus embryos to forskolin before vitrification. Theriogenology, 80:372-377.

Sanches BV, Lunardelli PA, Tannura JH, Cardoso BL, Pereira MHC, Gaitkoski G, Basso AC, Arnold DR, Seneda MM. 2016. A new direct transfer protocol for cryopreserved IVF embryos. Theriogenology, 85:1147-1151.

Saragusty J, Arav A. 2011. Current progress in oocyte and embryo cryopreservation by slow freezing and vitrification. Reproduction, 141: 1-19.

Segerson EC, Hansen TR, Libby DW, Randel RD, Getz WR. 1984. Ovarian and uterine morphology and function in Angus and Brahman cows. J Anim Sci,
59:1026-1046.

Silva-Santos KC, Santos GMG, Siloto LS, Hertel MF, Andrade ER, Rubin MIB, Sturion L, MeloSterza FA, Seneda MM. 2011. Estimate of the population of preantral follicles in the ovaries of Bos taurus indicus and Bos taurus taurus cattle. Theriogenology, 76:1051-1057.

Sreenan JM, Diskin MG. 1987. Factors affecting pregnancy rate following embryo transfer in the cow. Theriogenology, 27:99-113.

Stewart BM, Block J, Morelli P, Navarette AE, Amstalden M, Bonilla L, Hansen PJ, Bilby TR. 2011. Efficacy of embryo transfer in lactating dairy cows during summer using fresh or vitrified embryos produced in vitro with sex-sorted semen. J Dairy Sci, 94:3437-3445.

Stroud B. 2011. IETS 2011 Statistics and data retrieval committee report: the year 2010 worldwide statistics of embryo transfer in domestic farm animals. Embryo Trans Newslett, 29:14-23.

Stroud B. 2012. IETS 2012 statistics and data retrieval committee report: the year 2011 world wide statistics of embryo transfer in domestic farm animals. Embryo Trans Newslett, 30:16-26.

Sudano MJ, Paschoal DM, Rascado TD, Magalhaes LCO, Crocomo LF, Lima-Neto JF, LandimAlvarenga FD. 2011. Lipid content and apoptosis of in vitro-produced bovine embryos as determinants of susceptibility to vitrification. Theriogenology, 75:12111220.

Sudano MJ, Santos VG, Tata A, Ferreira CR, Paschoal DM, Machado R, Buratini J, Eberlin MN, Landim-Alvarenga FDC. 2012b. Phosphatidylcholine and sphingomyelin profiles vary in Bos taurus indicus and Bos taurus taurus in vitro-and in vivo-produced blastocysts. Biol Reprod, 87:1-11. doi: 10.1095/biolreprod.112.102897.

Sudano MJ, Paschoal DM, Maziero RRD, Rascado TS, Guastali MD, Crocomo LF, Magalhães LCO, Monteiro BA, Martins Jr A, Machado R, LandimAlvarenga FDC. 2013. Improving postcryopreservation survival capacity: an embryo-focused approach. Anim. Reprod, 10:160-167.

Sudano MJ, Paschoal DM, Rascado TS, Crocomo LF, Magalhaes LC, Junior AM, Machado R, Landim-Alvarenga FC. 2012a. Crucial surviving aspects for vitrified in vitro-produced bovine embryos. Zygote, 22:124-131.

Vajta G, Holm P, Kuwayama M, Booth PJ, Jacobsen H, Greve T, Callesen H. 1998. Open pulled straw (OPS) vitrification: a new way to reduce cryoinjuries of bovine ova and embryos. Mol Reprod Dev, 51:53-58.

Vajta G, Kuwayama M. 2006. Improving cryopreservation systems. Theriogenology, 65:236-244. Viana JHM. 2012. Levantamento estatístico da produção de embriões bovinos no Brasil em 2011: mudanças e tendências futuras. $O$ Embrião, 51:6-10.

Visintin JA, Martins JFP, Bevilacqua EM, Mello MRB, Nicácio AC, Assumpção MEOA. 2002. Cryopreservation of Bos taurus vs. Bos indicus embryos: are they really different? Theriogenology, 57:345-359.

Voelkel SA, Hu YX. 1992. Direct transfer of frozenthawed bovine embryos. Theriogenology, 37:23-37. 